\title{
Impact of cover change of land use on flood vulnerability in Jiangsu province
}

\author{
Shuangchen $\mathrm{Du}^{1, \mathrm{a}, \dagger}$ and Zichuan Zhang ${ }^{2, \mathrm{~b}, \uparrow}$ \\ ${ }^{1}$ Nanjing University of Information Science \& Technology, Nanjing, Jiangsu, 210044, China \\ ${ }^{2}$ Kang Chiao internation school, Kunshan, Jiangsu, 215300, China \\ †These authors contributed equally.
}

\begin{abstract}
In the context of global warming and rising sea levels, as urbanization continues to increase, the risk situation of urban systems facing floods has become more severe. Therefore, we constructed a vulnerability assessment model for urban flood disasters in Jiangsu Province, focusing on using GIS technology to classify the land use of each city in Jiangsu Province for supervised learning. We also established a flood disaster vulnerability model to evaluate the Vulnerability of 13 cities in Jiangsu Province. Evaluation and analysis of the changes in land use vulnerability use flood disasters in Jiangsu Province from 2000 to 2020. The results show that from 2000 to 2020, the Vulnerability to flooding disasters is on the rise as a whole. Yancheng has the lowest average Vulnerability to flooding disasters due to the low population density and high greening rate in the built-up area. On the other hand, Nantong City has the most heightened average flood vulnerability due to excessive population density. The low greening rate in built-up areas has led to a worsening of flood vulnerability. Suggestions have been made to reduce the Vulnerability of urban floods in Jiangsu Province, such as vigorously developing the local economy, reasonably adjusting the rate of urbanization, and building drainage infrastructure.
\end{abstract}

\section{Introduction}

Flood disasters are one of the leading natural disasters and are greatly affected by climate change. In the context of global warming, the temporal and spatial distribution of natural precipitation is gradually changing. Extreme precipitation events are becoming more frequent, the impact of flooding disasters is becoming wider and wider, and the losses to agricultural production are also increasing.

Jiangsu Province is located in the middle and lower reaches of the Yangtze River, in the mid-latitude zone on the east coast of the Asian continent. It belongs to the East Asian monsoon climate zone and is in the transition zone between subtropical and warm temperate climates. During the summer in June and July, affected by the East Asian monsoon, the area south of the Huaihe River enters the Meiyu period. The average annual precipitation during the Meiyu period is around $250 \mathrm{~mm}$ in most areas. Generally, about one week after the Jianghuai Meiyu begins, Huaibei region has entered the "Huaibei Rainy Season", when heavy rains frequently occur in the section and heavy rainfall is concentrated. Therefore, research on disaster vulnerability assessment of cities in Jiangsu Province is essential.

According to the IPCC report, Vulnerability refers to the degree of adverse effects of climate change, including climate variability and extreme climate events, on the system. It is a function of the characteristics, amplitude, and rate of change of climate change within the system and sensitivity and adaptability ${ }^{[1-3]}$. There are three types of existing evaluation methods that were been used for analyzing Vulnerability to flooding disasters. The first type is vulnerability evaluation based on historical disaster data. Through statistical analysis of historical flood disaster information, the Vulnerability of flood disasters can be evaluated. The second category is vulnerability assessment based on the indicator system. The Vulnerability is assessed by selecting indicators and determining the weighting method, and two indicators of natural factors and social factors are usually chosen for comprehensive analysis. The third category is vulnerability assessment based on scenario simulation analysis. They are combining historical data, given crucial influencing factors to construct disaster scenario models.

Regarding Vulnerability, different scholars have different opinions. Maskery defines Vulnerability as "the possibility of loss due to extreme events"[4]; Tobin and Montz determine Vulnerability as "potential loss"[5]; Deyle et al. believe that Vulnerability refers to "the sensitivity of human settlements to the impact of natural disasters." [6] Panizza interprets Vulnerability as "the total value of all people and things that exist in a given area that tend to lose due to natural disasters. ${ }^{[7]}$ Hewitt expands the breadth of vulnerability research to include nature, technology, and various fields of unnatural disasters and multiple links of disaster reduction ${ }^{[8]}$. Four factors

*Corresponding author's e-mail: a'Shuangchen.du@student.reading.ac.uk,

blakzhang@foxmail.com 
affecting the formation of disasters are proposed: Hazards, Vulnerability and adaptability, Intervening condition of danger, and Human coping and adjustments. Take comprehensive analysis and processing of various disaster factors from politics, economy, management, policy, and public participation to reduce disaster losses. Research on disaster vulnerability has gradually focused on human factors.

This paper considers the impact of six different types of land, including cultivated land, forest land, water area, construction land, unused land, and grassland. On the Vulnerability of flood disasters, combining the vulnerability parameters establishes comprehensive vulnerability evaluation models of various disasterbearing bodies (land-use types). The model compares the Vulnerability of 13 cities in Jiangsu Province regarding the flood disasters and the changes in Vulnerability of each town from 2000 to 2020 . This paper provides a basis for scientifically and rationally planning and sustaining the use of land resources in cities in Jiangsu Province by assessing the Vulnerability and studying the impact of changes in land use cover on the Vulnerability of cities in Jiangsu Province to flood disasters.

\section{Methods}

\subsection{Data collection}

In this study, the selected region is Jiangsu, China, and 13 prefecture-level cities are studied separately. The socioeconomic data used comes from 2000, 2010, and 2020 Jiangsu Statistical Yearbooks, including the administrative division of 15 prefecture-level cities in Jiangsu Province and the land-use area of each city from 2000 to 2020 . The remote sensing data source is mainly Landsat-30m images. The image has no or little cloud cover in the study area, the image quality is good, and the radiation correction and rough geometric correction have been carried out.

\subsection{Land use/cover classification based on GIS}

Many factors were considered to establish a land-use remote sensing classification system, including the image scale of remote sensing data, the level of resolution, the identifiability of features, the actual situation of the study area, and the practicability and systematicness of remote sensing images. Based on the actual condition in Jiangsu Province, referring to the classification system of "Classification of Land Use Status" (GB/T 21010-2007) and combining the temporal and spatial resolution and image characteristics of the data source, the land use and coverage types in the study area are divided into six categories of cultivated land, forest land, grassland, water area, construction land, and unused land.

The remote sensing image classification method in this study adopts supervised classification. That is, select the training sample area, calculate the spectral statistics of the actual area and combine with the field survey data of the study area, and perform the discrimination processing on the pixels of the entire image, which will have similar spectral characteristics. Furthermore, the pixels that meet specific discriminant rules were merged into one category, based on mathematical statistics theory, such as the maximum likelihood method, ISODATA.

\subsection{Vulnerability model based on land use}

According to $\mathrm{Fu}$ Xiaohong ${ }^{[9]}$, the economic vulnerability parameter table for flood disasters. This paper uses the analytic hierarchy process to calculate the flood vulnerability of 13 cities in Jiangsu Province. Each parameter is the economic vulnerability parameter of different disaster-bearing bodies: the weight of the impact of different land-use types on economic Vulnerability. The land-use types analyzed in this paper are cultivated land, forest land, water area, construction land, unused land, and grassland. Establish a comprehensive vulnerability assessment model for flood disasters of various land-use types in Jiangsu Province:

$$
\mathrm{T}=\sum_{i=1}^{n}(\alpha)_{i}+\beta_{i} * x_{i}(n=6)
$$

The comprehensive vulnerability assessment model for flood disasters in Jiangsu Province is as follows:

$$
\mathrm{T}=0.4289 x_{1}+0.1878 x_{2}+0.1933 x_{3}+1.2358 x_{4}+
$$
$0.0605 x_{5}+0.0268 x_{6}$

Among them, $x_{1}, x_{2}, x_{3, \ldots . .,} x_{6}$ are the cultivated land area, woodland area, water area, construction land area, unused land area, and grassland area in each city of Jiangsu province, respectively. This formula takes the total value of the economic vulnerability and population vulnerability parameters of flood disasters as the weight of each category on the Vulnerability of flood disasters, reflecting the extent of the impact of changes in each land type on the Vulnerability of flood disasters.

\section{Results and discussion}

\subsection{Remote sensing image classification results}

After the classification of remote sensing images and the correction of the 2005-2020 Jiangsu Provincial Statistical Yearbook, the remote sensing image classification data of Jiangsu Province in 2000, 2010, and 2020 can be obtained. The Arcmap10.0 computer-aided mapping software produces the land use/cover classification results map in ArcGIS10.0.

During 2000-2020, various land use covers in Jiangsu Province has changed to varying degrees. The most considerable change in this area was cultivated land, followed by construction land, and then unused land and forest land. The area of grassland and water area changed slightly. Cultivated land, grassland, and unused land decreased, while construction land, woodland, and water increased. Details as follows: 


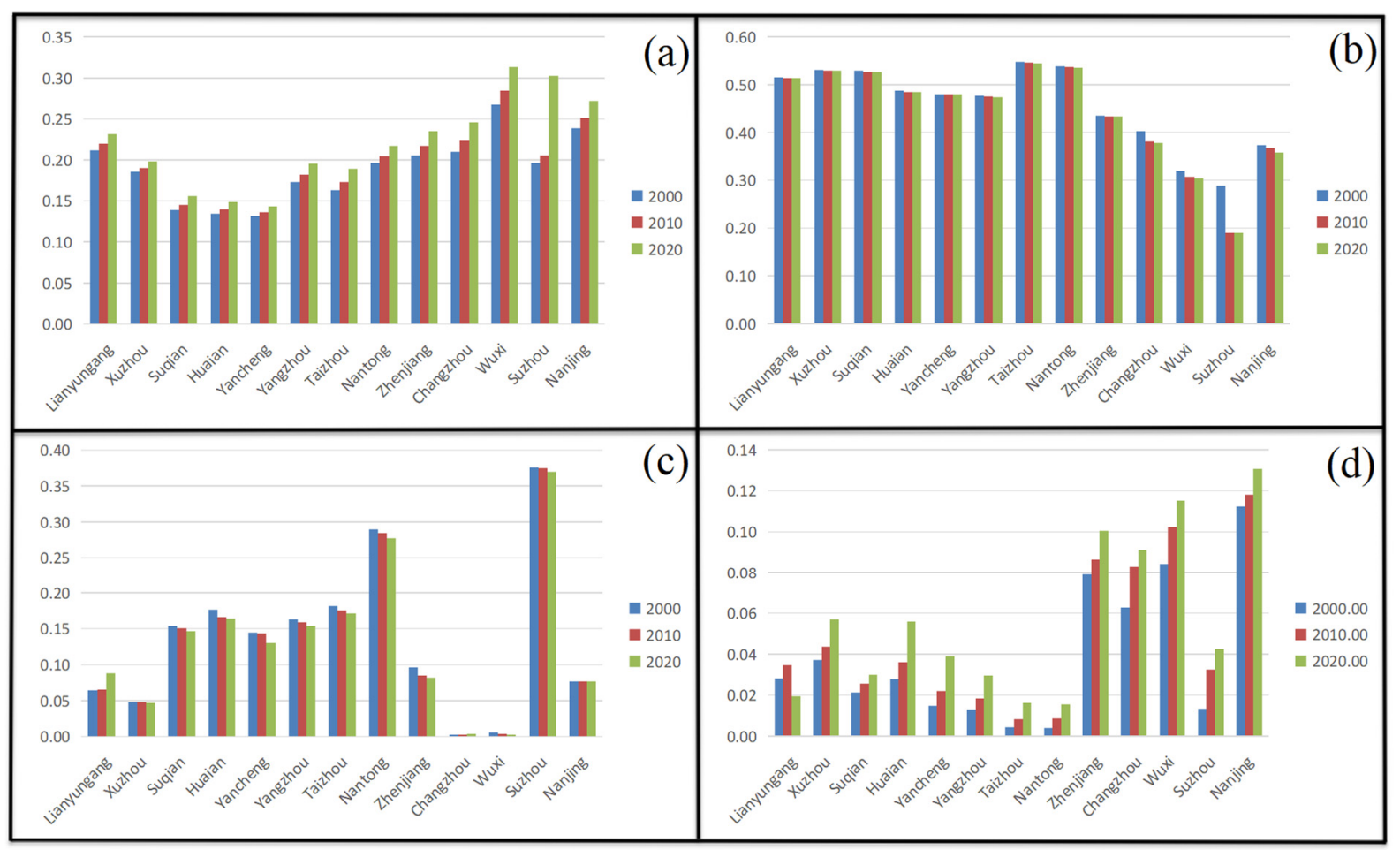

Fig. 1 Changes in the proportion of land use in cities of Jiangsu Province in 2000, 2010, and 2020:(a) Changes in the proportion of land use for construction, (b) Changes in the proportion of arable land, (c) Changes in the proportion of water area, (d) Changes in the proportion of forestland

Figure 1(a) shows that the overall increase is year by year in construction land. From 2000-2010, Wuxi City was the city with the most significant proportion of construction land area, and Yancheng City was the city with the least proportion of construction land area. There was a decline between 2010 and 2020 in forest land except for Lianyungang City, while the area of forest land in the rest of the cities increased in the first year. From 2000 to 2020, Nanjing was the city with the largest forest land, and Taizhou was the city with a minor proportion of forest land. From Figure 1(b), the overall cultivated land is slowly decreasing year by year. From 2000 to 2020, Taizhou City was the city with the largest proportion of arable land, and Suzhou City was the city with a minor proportion of arable land. In terms of grassland, all cities have fewer grassland areas, and the grassland area in Zhenjiang City is significantly larger than that in other cities. And each city shows a decreasing trend from 2000 to 2020. In terms of unused land, all cities have decreased. From 2000 to 2020, Yancheng City has the largest proportion of unused land, and Wuxi City has the least amount of unused land. In terms of waters, except for Lianyungang City, the overall decrease is decreasing year by year. From Figure 1(c), from 2000 to 2020, Suzhou City has the most significant water area, and Changzhou City has the smallest water area.

The three major driving forces of natural factors, socio-economic factors, human activities of land use and cover change the three interact and develop together as a driving force to promote land use and cover changes. They are manifested explicitly as changes in the natural environment, social and economic development, and human changes in values. Under the action of various driving forces, land use has undergone dynamic changes, and consequently, the purpose and methods of land use have also changed. Compared with 2010 and 2000, other land types have shifted to construction land and water areas, and the two land types have increased in proportion. Compared with 2010 and 2020, different land types have shifted to construction land and water areas, and construction land has increased.

From 2000 to 2020, with the development of the social economy and the continuous increase of population, construction land has shown a continuously increasing trend. The proportion of cultivated land and unused land transferred to construction land is relatively large. The increase in construction land is in line with the law of social development. However, although construction land continues to increase, it cannot meet people's growing social demand for construction land.

Urbanization has changed the topography, geomorphology, and geological conditions. The impervious area of urban areas has increased, resulting in additional runoff generation capacity. Under heavy rain conditions, more rainfall will be converted into a surface runoff with flow and energy concentration, which has increased the erosion capacity of water flow. Resulting in intensified soil erosion, soil erosion, and a large amount of sedimentation, causing flood drainage ditches, sewers, rivers, and other facilities to discharge. The flood discharge capacity is reduced. River remediation, canalization, hardening of river banks, straightening of bends, river banks to withstand floods. Reinforcement has improved the flood-carrying ability of the river and met the needs of shipping, but it has severely damaged the natural form of the river and cut off the river. The flow is connected with the surrounding environment. The buffer zone caused by the development of the shore zone is 
compressed or even disappeared, and the lateral movement space of the water is sharply reduced.

These factors all lead to reduced morphological heterogeneity, simplification of habitats, ecological degradation, and the gradual loss of the original functions of rivers. Furthermore, with extreme and concentrated rainfall gradually increasing and the water permeability of the underlying surface of the city reduced, more rainfall is discharged into the river in the form of rain and flood runoff. It causes river flood disasters to become more frequent and increasing in intensity, and even directly lead to waterlogging. Especially in urban areas where the population and property are highly concentrated, and the population density increases, the impact of flooding is more pronounced.

\subsection{Vulnerability Assessment of Land Use Flooding in Jiangsu Province}

This paper analyzes the Vulnerability of flood disasters based on the current land use data in 2000, 2010, and 2020 in 13 cities in Jiangsu Province. The vulnerability analysis concluded that the land use flood disasters between different cities in Jiangsu Province The difference and connection invulnerability, by drawing the annual land use vulnerability map of each city, visually show the relative size of the land use vulnerability cities.

The Jiangsu Province studied includes 13 cities with a total land area of $10.700,000$ hectares. The vulnerability values of flood disasters were calculated based on the established comprehensive vulnerability model of land use flood disasters and the statistical land-use data of each city in 2000,2010 , and 2020 .

Table 1 Changes of flood vulnerability value of land use in each city from 2000 to 2020

\begin{tabular}{cccc}
\hline City & $\mathbf{2 0 0 0}$ & $\mathbf{2 0 1 0}$ & $\mathbf{2 0 2 0}$ \\
\hline Lianyungang & $5.01 \times 10^{3}$ & $5.11 \times 10^{3}$ & $5.27 \times 10^{3}$ \\
Xuzhou & $4.79 \times 10^{3}$ & $4.81 \times 10^{3}$ & $4.92 \times 10^{3}$ \\
Suqian & $4.32 \times 10^{3}$ & $4.38 \times 10^{3}$ & $4.52 \times 10^{3}$ \\
Huaian & $4.15 \times 10^{3}$ & $4.20 \times 10^{3}$ & $4.35 \times 10^{3}$ \\
Yancheng & $4.03 \times 10^{3}$ & $4.04 \times 10^{3}$ & $4.18 \times 10^{3}$ \\
Yangzhou & $4.53 \times 10^{3}$ & $4.64 \times 10^{3}$ & $4.82 \times 10^{3}$ \\
Taizhou & $4.72 \times 10^{3}$ & $4.83 \times 10^{3}$ & $5.04 \times 10^{3}$ \\
Nantong & $5.32 \times 10^{3}$ & $5.40 \times 10^{3}$ & $5.55 \times 10^{3}$ \\
Zhenjiang & $4.74 \times 10^{3}$ & $4.87 \times 10^{3}$ & $5.11 \times 10^{3}$ \\
Changzhou & $4.45 \times 10^{3}$ & $4.56 \times 10^{3}$ & $4.84 \times 10^{3}$ \\
Wuxi & $4.84 \times 10^{3}$ & $5.04 \times 10^{3}$ & $5.40 \times 10^{3}$ \\
Suzhou & $4.42 \times 10^{3}$ & $4.14 \times 10^{3}$ & $5.34 \times 10^{3}$ \\
Nanjing & $4.92 \times 10^{3}$ & $5.06 \times 10^{3}$ & $5.29 \times 10^{3}$ \\
\hline
\end{tabular}

On the whole, different cities have different vulnerability values due to different natural resources and location differences. According to the vulnerability value of each city, the urban vulnerability value of Jiangsu Province is divided into four vulnerable cities. Among them, cities with high Vulnerability have a vulnerability value greater than or equal to 5000). Cities with the second-highest Vulnerability have a vulnerability value between 4700 to 5000). Cities with medium Vulnerability have vulnerability values between 4300 to 4700 .

In comparison, low vulnerability cities have vulnerability values less than 4300 . Specifically, Nantong, Wuxi, Lianyungang, Nanjing are highly vulnerable. Xuzhou, Yangzhou, Taizhou, and Zhenjiang are the second most vulnerable cities, Suzhou City, Suqian City, and Changzhou City are medium-vulnerability cities, Huai'an City and Yancheng City are low-vulnerability cities.

Therefore, the following suggestions and countermeasures are proposed to reduce the Vulnerability of urban floods. First, minimize urban flood vulnerability by promoting local economic development. Strong economic conditions are the prerequisite for urban disaster relief work ${ }^{[10]}$. When encountering flood disasters, if the city does not have a certain financial foundation, it will be hit by flood disasters. Among the 13 cities, the GDP per capita of Suqian is relatively low. Therefore, the decisionmaker should adjust the industrial structure and spatial layout, meanwhile formulating related economic policies. Second, rationally to promote the high-quality development of the regional economy ${ }^{[11]}$.

Reasonably adjust the speed of urbanization to reduce the vulnerability of cities to floods. The process of urbanization will have a series of impacts on cities ${ }^{[12]}$. For example, the expansion of urban areas leads to a decrease in soil area, severely weakening the soil's ability to conserve water and is not conducive to infiltration. The density of the road network and the density of drainage pipes greatly impact the Vulnerability of urban floods and water logging ${ }^{[13]}$. The greening rate of built-up areas has always maintained a stable weight, which has a certain influence on urban water-logging prevention. The focus of urbanization adjustment is to optimize the structure of land resource utilization. Cultivated land resources significantly impact the Vulnerability of flood-desert disasters, and it is vulnerable to threats and damages from flood-desert disasters. Therefore, when formulating land plans, it is necessary to ensure the basic farmland does not decrease, and the land converted from farmland to forests and grass does not rebound ${ }^{[14]}$. Meanwhile, fully grasp the rules of the transfer of various types of land, in which the government must guide the land to high-yield, highquality, and efficient according to the conversion rules. Therefore, achieving in-depth development and formulate feasible land-use adjustment plans ${ }^{[15]}$. Realize sustainable use of land resources, reduce the Vulnerability of flood port disasters; implement the "returning people without returning farmland" to the lake, and reclaim the flood storage areas ${ }^{[16]}$. The population has to move into a safe area, and the land in the reclamation area is planted and raised when the water is low and flooded when the water is high. To improve the land utilization rate, the government has to focus on adjusting the land use structure of the hilly land, protecting and rebuilding the vegetation in the mountainous area to form a water and soil conservation land use pattern ${ }^{[17]}$.

Optimize the urban population structure and strengthen relevant disaster education to reduce urban 
flood vulnerability ${ }^{[18]}$. On the one hand, through policy guidance, strengthen the management of the migrant population, implement the registration of migrant population information, and continuously optimize the population structure ${ }^{[19]}$. On the other hand, it improves the public's awareness and ability to prevent flood disasters and popularize disaster knowledge in schools, communities, and other places. Thus, enhance awareness of risk prevention and promote public participation in urban flood prevention emergency management ${ }^{[20]}$. Among the 13 cities, the population density of Wuxi is relatively high, so the population should be controlled reasonably to achieve sustainable development of population and economy.

\section{Conclusion}

Through the analytic hierarchy process, the urban flood vulnerability assessment model is constructed. The vulnerability parameters of various disaster-bearing bodies are different. The comprehensive vulnerability parameters of cultivated land, forest land, water area, construction land, unused land, and grassland are 0.4289 , $0.1878,0.1933,1.2358,0.0605$, and 0.0268 , reflecting different lands. The difference in the impact of utilization types on the Vulnerability of flood disasters, of which construction land has an immense impact on the Vulnerability of flood disasters, followed by cultivated land, water, and woodland have the same impact on Vulnerability to floods. In contrast, unused land and grassland have the same effects on flood vulnerability. The effect of disaster vulnerability is relatively tiny.

The Vulnerability of flood disasters in Jiangsu Province is divided into low Vulnerability, second-lowest Vulnerability, medium vulnerability, second-highest Vulnerability, and high Vulnerability. The Vulnerability of flood disasters in Jiangsu Province presents a specific spatial pattern. Xuzhou and Yancheng are highly vulnerable cities, Nantong, Suzhou, and Huai'an are the second most susceptible cities, Lianyungang, Suqian, and Nanjing Yangzhou and Yangzhou are mediumvulnerability cities. At the same time, Taizhou, Wuxi, Changzhou, and Zhenjiang are low-vulnerability cities. Furthermore, the Vulnerability of each city shows an increasing trend over time. From 2000 to 2020, the Vulnerability of each city to flood disasters continues to increase from the perspective of individual cities. According to the comprehensive land-use planning data, the annual vulnerability value of each city is calculated to be greater than its Vulnerability in the previous years. The vulnerability value of each city shows an upward trend with the growth of time. Among them, the vulnerability value of construction land has the most considerable change and the fastest growth, followed by woodland.

This paper uses the relevant indicators of different disaster-bearing bodies (land-use types) to construct a flood vulnerability assessment system and conduct quantitative research. It is conducive to discovering the relationship between different indicators and the degree of urban flood disasters and enhances the comparability of different regions. At present, limited to data availability, the indicators included in the indicator system are limited. If the dynamic collection and sharing of primary flood control data and storm disaster data can be optimized, the evaluation model can be further improved. At the same time, this evaluation model can be used to evaluate and compare different types of disasters in the future.

\section{References}

1. WOLF T, MCGREGOR G. The development of a heat wave vulnerability index for London, United Kingdom [J]. Weather and Climate Extremes, 2013, 1: 59-68.

2. ZHU Q, LIU T, LIN H, et al. The spatial distribution of health vulnerability to heat waves in Guangdong Province, China [J]. Global Health Action, 2014, 7(1): 25051.

3. EL-ZEIN A, TONMOY F N. Assessment of vulner ability to climate change using a multi-criteria outranking approach with application to heat stress in Sydney [J]. Eco-logical Indicators, 2015, 48: 207-217.

4. Maskrey. Disaster Mitigation[M]. Oxford: Oxfam, 1989.1-100.

5. Tobin \& Montz. Natural Hazards: Explanation and Integration [M]. New York:TheGuilfordPress, 1997.1338.

6. Deyle, French, Olshansky, Hazard assessinent: the factual basis for planning and mitigation[A]. Cooperating with Nature: Confronting Natural Hazards with Land Use Planning for Sustainable Communities[C]. 1998.119-166.

7. Panizza. Environmental Geomorphology[M]. Amsterdam: Elsevier, 1996.1-268.

8. Hewit. Region of Risk[M]. Singapore: Longman Singapore Pubisher (Pte) Ltd, 1997.

9. Fu Xiaohong. Analysis of land use / cover change and its impact on flood vulnerability in Dongting Lake area [D]. Hunan Normal University, 2014

10. Li Zhenxing. Causes and Countermeasures of urban flood disaster crisis: a case study of Nanjing [J]. Journal of Guilin municipal Party school, 2018,18 (02): 62-65

11. Wang Qun, Yu Liuyue, Wu zhaodan, Chen Haoran. Vulnerability assessment of urban flood in Yangtze River Economic Belt: a case study of Nanjing, Wuhan and Chengdu [J]. Water economy, 2020,38 (03): 55$61+85-86$

12. Cheng Xiaotao, Han song, Wang Hongtao, Liao Jingxia. Discussion on flood control and disaster reduction in the new era $[\mathrm{J}]$. China water conservancy, 2012 (11): 43-46

13. Zhang Han. Impact of climate change and urbanization on urban flood disaster risk in Pearl River Delta [D]. South China University of technology, 2019

14. Liu Jianfen, Wang Huimin, Zhang Xingnan. Causes and Countermeasures of frequent urban flood disasters under the background of urbanization [J]. 
Journal of Hohai University (PHILOSOPHY AND SOCIAL SCIENCES EDITION), 2012,14 (01): 73$75+92$

15. Deng Jinyun, Liu Congcong. Risk and loss assessment of urban waterlogging under different land use types [J]. Journal of safety and environment, 2018,18 (06): 2293-2299

16. Sun dianchen, Wang Huimin, Huang Jing, Liu Gaofeng. Urban flood disaster risk and land type adjustment strategy in Poyang Lake Basin: a case study of Jingdezhen City [J]. Resources and environment of Yangtze River Basin, 2018,27 (12): 2856-2866

17. Zhang Guoping, Lu houquan, Wang Jianlin. Assessment of the impact of rainstorm and flood on agricultural production and ecological environment in the Huaihe River Basin by remote sensing technology [a]. China Meteorological Society. Innovation of meteorological science and technology and development of Atmospheric Science in the new century: Proceedings of the annual meeting of China Meteorological society 2003 "hydrological and Meteorological Problems of Huaihe River flood on March 7" [C]. China Meteorological Society: China Meteorological Society, 2003:5

18. Huang Jing, she Jingwen. Vulnerability assessment and influencing factors analysis of flood disaster in urban agglomerations of Yangtze River Delta [J]. Journal of Hohai University (PHILOSOPHY AND SOCIAL SCIENCES EDITION), 2020,22 (06): 39$45+110-111$

19. Wang Hao. Some understandings on urban flood control in China under the new situation [J]. Flood control and drought relief in China, 2019,29 (08): 1-2

20. Zhang Liru, he Yonghui, Wang Yan, Li Hui, Dai Jiaqi. Study on the status quo of urban flood monitoring and flood control countermeasures in China [a]. Water Resources Professional Committee of Chinese society of natural resources, hydrogeological Professional Committee of Chinese geographic society, water resources professional committee of Chinese hydraulic society, hydrogeological professional Committee of Chinese hydraulic society Water Issues Committee of China Society for sustainable development, Shenzhen Association for science and technology, Nanshan District Association for science and technology of Shenzhen. Innovative research on water system for global change: Proceedings of the 15th China Water Forum [C] Water resources professional committee of China Society of water resources, hydrology Professional Committee of China Society of water resources, water issues Professional Committee of China Society for sustainable development, Shenzhen Science and Technology Association, Nanshan District Science and Technology Association of Shenzhen: China water resources and Hydropower Press, 2017:4 